\title{
Investigation of free vibrations of three- layered cylindrical shell supported by transverse ribs
}

\author{
Mykola Surianinov ${ }^{\# 1}$, Tetiana Yemelianova ${ }^{* 2}$, Dina Lazarieva ${ }^{* 3}$ \\ "Department of applied mathematics, \\ Odessa State Academy of Civil Engineering and Architecture, Odessa, Ukraine \\ ${ }^{1}$ sng@ogasa.org.ua \\ *Department of physic and general engineering disciplines, \\ Kherson State Agriculture University, Kherson, Ukraine \\ 2e.tatyana.2014@ukr.net \\ *Department of structural mechanics, \\ Odessa State Academy of Civil Engineering and Architecture, Odessa, Ukraine \\ 3dvl7@ukr.net
}

\begin{abstract}
Construction of calculation model and development of algorithm of free vibrations investigation in three-layered cylindrical shell with light-weight aggregate, supported by transverse ribso are considered in paper. Variation equation of transverse vibrations of three-layered shell of symmetric structure, supported by ribs in two perpendicular directions, taking into account the action of longitudinal forces in meddle surfaces of external layers and ribs is achieved. For external bearing layers of shell there are accepted hypotheses of Kirchgoff-Love and for aggregate there is accepted the linear law of tangential displacements change by thickness. Aggregate transverse deformations was not taken into account. The hypotheses of Bernoulli were accepted for ribs. There was taken into account only bending of ribs in vertical surface. Using passage to the limit there was achieved conditions on ribs lines without taking into account shear deformations in ribs. There are given values of parameter of free vibrations first frequency of three-layered sloping cylindrical shell with light-weight aggregate, supported by one and three transverse ribs.
\end{abstract}

Keyword - Three-layered shell, Light-weight aggregate, Free vibrations, Ribs, Displacement function, Force function, Frequency equation, First vibration frequency parameter

\section{INTRODUCTION}

Layered constructions, in particular three-layered shells, are widely used in many industries, including industrial and civil construction.

Three-layered shell consists of two comparatively thin external layers of strong material between which comparatively thick layer of material with low strength and light volume weight is placed. External layers are called bearing and internal layer is called aggregate.

Three-layered constructions are classified by the type of aggregate, bearing layers' material, connection type. Light-weight aggregates can be styrofoam, honeycomb core, corrugations (single or double), tubular aggregate, etc.

Three-layered construction application allows to increase the shell stability, reduce the negative influence of initial deflections on stability, use mechanical properties of material in better way.

\section{PROBLEM FORMULATION}

Forsuch kind of constructions, the solution of free vibrations problem has fundamental importance because it allows to solve many other problems of dynamics. A special role has information about the first frequency of free vibrations. The problem of three-layered shell without support by ribs was considered by a lot of authors. Let's note the works that have already become classical [1-4], as well as later publications [5-9]. There are no too much publications devoted to vibrations of three-layered shells supported by ribs [10-12].

\section{A. Aim of paper}

The aim of the work is to build a computational model and to develop an algorithm for studying the free vibrations of a three-layer cylindrical shell with a lightweight aggregate that is supported by transverse ribs with edges simple support. 


\section{B. Materials and methods}

Using the functional-action by Ostrogradskiy-Hamilton, there is received variation equation of transverse vibrations of three-layered shell of symmetric construction, that is supported by ribs in two perpendicular directions, taking into account the action of longitudinal forces in middle planes of external layers and in ribs.

For external bearing layers of shell there are accepted hypotheses of Kirchhoff-Love and for aggregate there is accepted the linear law of tangential displacement on thickness change. Transverse deformations of aggregate was not taken into account. For plates ribs the hypotheses of Bernoulli was accepted. Only ribs bending in vertical plane was taken into account. With the help of the limit transition, conditions are obtained for the ribs lines without considering the shear deformations in the ribs [3].

\section{RESEARCH RESULTS}

Let's consider free vibrations of three-layered sloping cylindrical shell with light-weight transverse-isotropic aggregate, that is supported by transverse ribs. Distance between ribs and ribs stiffness we will consider as equal (Fig. 1).

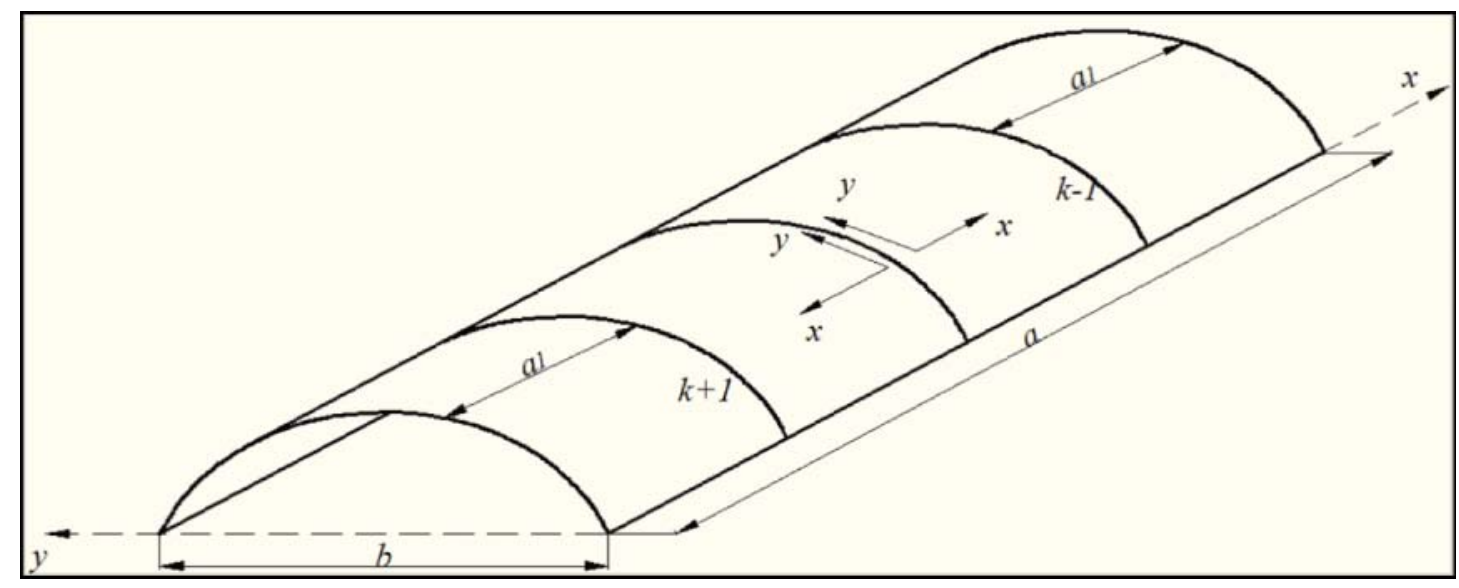

Fig. 1. Three-layered shell supported by ribs scheme

Differential equations of flexural vibrations of shell area that is located between ribs [10], has a form:

$$
\begin{aligned}
& \nabla^{4} \Phi+\frac{\bar{B}}{R} \frac{\partial^{2}}{\partial x^{2}}\left(\varphi-\frac{B h}{G} \nabla^{2} \varphi\right)=0 \\
& \nabla^{4} \varphi+\frac{1}{\hat{A} D^{*}} \frac{\partial^{2} \Phi}{\partial x^{2}}-m_{\sigma \sigma} \omega^{2}\left(\varphi-\frac{B h}{G_{3}} \nabla^{2} \varphi\right)=0 ; \\
& \Psi-\frac{1-\mu}{2 G_{3}} B h \nabla^{2} \Psi=0 .
\end{aligned}
$$

In work [11] by the way of introduction of displacement function $F(x, y)$ it is performed the simplification of differential equation (1) - (3) system and there is get the resolving equation of free vibrations:

$$
\nabla^{4} \nabla^{4} F+\frac{\bar{B}}{R^{2} D} \frac{\partial^{4}}{\partial x^{4}}\left(1-\frac{B h}{G_{3}} \nabla^{2}\right) F-\frac{\omega^{2} m_{o \sigma}}{D^{*}}\left(1-\frac{B h}{G_{3}} \nabla^{2}\right) F \nabla^{4} F=0
$$

In equations $(1)-(4)$ there are accepted symbols:

$$
\begin{aligned}
& B=\frac{E \delta}{1-\mu^{2}} ; D=\frac{E \delta}{12\left(1-\mu^{2}\right)} ; D^{*}=2 B H^{2} ; H=h+0,5 \delta ; \\
& m_{\omega}=\frac{m_{\text {об }} \cdot \omega \cdot b^{2}}{\pi^{2} B D^{*} \alpha_{n}^{2}} ; m_{\text {об }}=2\left(\rho_{H} \cdot \delta+\rho_{3} \cdot h\right) ; \varphi=\nabla^{4} F ; \\
& \Phi=-\frac{\bar{B}}{R} \frac{\partial^{2}}{\partial x^{2}}\left(1-\frac{B h}{G_{3}} \nabla^{2}\right) F ;
\end{aligned}
$$

$\delta$ - external layers thickness, $2 h$ - thickness of external layers and aggregate. 
Solution of equations (3) and (4) for area of shell between two adjacent ribs we will find in the form:

$$
F=f_{1}(x) \sin \frac{\pi}{b} y ; \Psi=f_{2}(x) \cos \frac{\pi}{b} y .
$$

Substituting (5) into (3) and (4), we obtain

$$
\begin{aligned}
& F=\sin \frac{\pi y}{b}\left[\cos x \varphi_{1}\left(C_{1}+C_{3}\right)+\sin x \varphi_{1}\left(C_{2}-C_{4}\right)\right]\left[\rho_{1}^{x}+\left(\cos x \varphi_{2}\left(C_{5}+C_{7}\right)+\sin x \varphi_{2}\left(C_{6}-C_{8}\right)\right)+\rho_{2}^{x}\right] ; \\
& \Psi=\left(C_{9} \cos [\beta x]+C_{10} \sin [\beta x]\right) \cos \left[\frac{\pi}{b} y\right] .
\end{aligned}
$$

Here $\tan \varphi_{1}=\frac{r}{s} ; \tan \varphi_{2}=\frac{d}{c} ; \rho_{1}=\left|\sqrt{s^{2}+r^{2}}\right| ; \rho_{2}=\left|\sqrt{c^{2}+d^{2}}\right|$, where $s, c-$ real, $r, d-$ complex roots of characteristic equation

$$
\begin{aligned}
& \lambda^{8}+k_{0}\left(m_{\omega}-\frac{\pi^{2} \alpha^{2}}{b^{2}}\right) \lambda^{6}+\frac{\pi^{4}}{b^{4}}\left(\pi^{2}\left(2+\alpha^{2}\right)-b^{2} m_{\omega}+k_{0}\left(\pi^{2} \alpha^{2}-b^{2} m_{\omega}\right)\right) \lambda^{4}+ \\
& +\frac{\pi^{4}}{b^{4}} k_{0} m_{\omega} \lambda^{2}+\frac{\pi^{6}}{b^{8}}\left(\pi^{2}-b^{2}\left(1+k_{0}\right) m_{\omega}\right)=0,
\end{aligned}
$$

where $k_{0}=\frac{\pi^{2} B h}{G_{3} b^{2}} ; \alpha^{2}=\frac{\bar{B} b^{4}}{R^{2} D^{*} \pi^{4}}$.

Assuming that diaphragms are installed on the edges of the shell, the boundary conditions for the case of free support will be written in the form:

$$
\begin{array}{ll}
\text { at } x=0 & w=\frac{\partial u_{\beta}}{\partial x}=v \beta=v_{\alpha}=\frac{\partial^{2} \Phi}{\partial y^{2}}=0, \\
\text { at } y=0 & w=\frac{\partial v_{\beta}}{\partial y}=u \beta=u_{\alpha}=\frac{\partial^{2} \Phi}{\partial x^{2}}=0 .
\end{array}
$$

Assuming for every area its coordinate axes[12], we will locate them in the start of area and denote $f_{1}(x)$ in the starts and in the ends of area (at $x=0$ and $x=a_{1}$, where $a_{1}=a / m, m$ - number of areas) as $\eta_{k}$ and $\eta_{k+1}$; value $f_{1}^{I I}(x)$ as $\mu_{k}$ and $\mu_{k+1}$; value $f_{1}^{I V}(x)$ as $\varsigma_{k}$ and $\varsigma_{k+1}$; value $f_{1}^{V I}(x)$ as $\zeta_{k}$ and $\zeta_{k+1}$; value $f_{1}^{V I I I}(x)$ as $\varphi_{k}$ and $\varphi_{k+1}$.

Using these conditions, we express through them values of arbitrary constant $C_{i}$ of solutions (6) and (7), which are determined from the system of equations (11):

$$
\begin{aligned}
& \eta_{k}=C_{1}^{k}+C_{3}^{k}+C_{5}^{k}+C_{7}^{k} \\
& \mu_{k}=\left(C_{1}^{k}+C_{3}^{k}\right)\left(p_{5}^{2}-\varphi_{1}^{2}\right)+\left(C_{5}^{k}+C_{7}^{k}\right)\left(p_{6}^{2}-\varphi_{2}^{2}\right)+2\left(C_{2}^{k}-C_{4}^{k}\right) p_{5} \varphi_{1}++2\left(C_{6}^{k}-C_{8}^{k}\right) p_{6} \varphi_{2}, \\
& \varsigma_{k}=\left(C_{1}^{k}+C_{3}^{k}\right)\left(p_{5}^{4}-6 p_{5}^{2} \varphi_{1}^{2}+\varphi_{1}^{4}\right)+\left(C_{5}^{k}+C_{7}^{k}\right)\left(p_{6}^{4}-6 p_{6}^{2}+\varphi_{2}^{4}\right)+ \\
& +\left(C_{2}^{k}-C_{4}^{k}\right)\left(4 p_{5}^{3} \varphi_{1}-4 p_{5} \varphi_{1}^{3}\right)+\left(C_{6}^{k}-C_{8}^{k}\right)\left(4 p_{6}^{3} \varphi_{2}-4 p_{6} \varphi_{2}^{3}\right) ; \\
& \xi_{k}=\left(C_{1}^{k}+C_{3}^{k}\right)\left(p_{5}^{6}-15 p_{5}^{4} \varphi_{1}^{2}+15 p_{5}^{2} \varphi_{1}^{4}-\varphi_{1}^{6}\right)+\left(C_{5}^{k}+C_{7}^{k}\right)\left(p_{6}^{6}-15 p_{6}^{4} \varphi_{2}^{2}+15 p_{6}^{2} \varphi_{2}^{4}-\varphi_{2}^{6}\right) ; \\
& \varphi_{k}=-\beta^{2} C_{9} ; \\
& \eta_{k+1}=\left(\left(C_{1}^{k+1}+C_{3}^{k+1}\right) p_{1}+\left(C_{2}^{k+1}-C_{4}^{k+1}\right) p_{2}\right) \rho_{1}^{a_{1}}+\left(\left(C_{5}^{k+1}+C_{7}^{k+1}\right) p_{3}+\left(C_{6}^{k+1}-C_{8}^{k+1}\right) p_{4}\right) \rho_{2}^{a_{2}} ; \\
& \mu_{k+1}=\left(C_{1}^{k+1}+C_{3}^{k+1}\right)\left(\rho_{1}^{a_{1}}\left(p_{1}\left(p_{5}^{2}-\varphi_{1}^{2}\right)-2 p_{2} p_{5} \varphi_{1}\right)\right)+\left(C_{2}^{k+1}-C_{4}^{k+1}\right)\left(\rho_{1}^{a_{1}}\left(p_{2}\left(p_{5}^{2}+\varphi_{1}^{2}\right)+2 p_{1} p_{5} \varphi_{1}\right)\right)+ \\
& +\left(C_{5}^{k+1}+C_{7}^{k+1}\right)\left(\rho_{2}^{a_{1}}\left(p_{3}\left(p_{6}^{2}-\varphi_{2}^{2}\right)-2 p_{4} p_{6} \varphi_{2}\right)\right)+\left(C_{6}^{k+1}+C_{8}^{k+1}\right)\left(\rho_{2}^{a_{1}}\left(p_{4}\left(p_{6}^{2}-\varphi_{2}^{2}\right)+2 p_{3} p_{6} \varphi_{2}\right)\right) ;
\end{aligned}
$$




$$
\begin{aligned}
& \varsigma_{k+1}=\left(C_{1}^{k+1}+C_{3}^{k+1}\right)\left(\rho_{1}^{a_{1}}\left(p_{1}\left(p_{5}^{4}-6 p_{5}^{2} \varphi_{1}^{2}+\varphi_{1}^{4}\right)-4 p_{2} p_{5} \varphi_{1}\left(p_{5}^{2}+\varphi_{1}^{2}\right)\right)\right)+ \\
& +\left(C_{2}^{k+1}-C_{4}^{k+1}\right)\left(\rho_{1}^{a_{1}}\left(p_{2}\left(p_{5}^{4}+6 p_{5}^{2} \varphi_{1}^{2}+\varphi_{1}^{4}\right)+4 p_{1} p_{5} \varphi_{1}\left(p_{5}^{2}-\varphi_{1}^{2}\right)\right)\right)+ \\
& +\left(C_{5}^{k+1}+C_{7}^{k+1}\right)\left(\rho_{2}^{a_{1}}\left(p_{3}\left(p_{6}^{4}-6 p_{6}^{2} \varphi_{2}^{2}+\varphi_{2}^{4}\right)-4 p_{4} p_{6} \varphi_{2}\left(p_{6}^{2}+\varphi_{2}^{2}\right)\right)\right)+ \\
& +\left(C_{6}^{k+1}+C_{8}^{k+1}\right)\left(\rho_{2}^{a_{1}}\left(p_{4}\left(p_{6}^{4}+6 p_{6}^{2} \varphi_{2}^{2}+\varphi_{2}^{4}\right)+4 p_{3} p_{6} \varphi_{2}\left(p_{6}^{2}-\varphi_{2}^{2}\right)\right)\right), \\
& \xi_{k+1}=\left(C_{1}^{k+1}+C_{3}^{k+1}\right)\left(\rho_{1}^{a_{1}}\left(p_{2} p_{5}\left(p_{5}^{5}-6 p_{5}^{4} \varphi_{1}-20 p_{5}^{2} \varphi_{1}^{3}-6 \varphi_{1}^{5}\right)+p_{2}\left(p_{5}^{6}-15 p_{5}^{4} \varphi_{1}^{2}+15 p_{5}^{2} \varphi_{1}^{4}-\varphi_{1}^{6}\right)\right)\right)+ \\
& +\left(C_{2}^{k+1}-C_{4}^{k+1}\right) \cdot\left(\rho_{1}^{a_{1}} \varphi_{1}\left(p_{2} \varphi_{1}\left(15 p_{5}^{4}+15 p_{5}^{2} \varphi_{1}-\varphi_{1}^{4}\right)+p_{1}\left(6 p_{5}^{5}-20 p_{5}^{3} \varphi_{1}^{2}+6 p_{1} \varphi_{1}^{4}\right)\right)\right)+ \\
& ++\left(C_{5}^{k+1}+C_{7}^{k+1}\right)\left(\rho_{2}^{a_{1}}\left(p_{4} p_{6}\left(p_{6}^{5}-6 p_{6}^{4} \varphi_{2}-20 p_{6}^{2} \varphi_{2}^{3}-6 \varphi_{2}^{5}\right)+p_{4}\left(p_{6}^{6}-15 p_{6}^{4} \varphi_{2}^{2}+15 p_{6}^{2} \varphi_{2}^{4}-\varphi_{2}^{6}\right)\right)\right)+ \\
& +\left(C_{6}^{k+1}+C_{8}^{k+1}\right) \cdot\left(\rho_{2}^{a_{1}} \varphi_{2}\left(p_{3} \varphi_{2}\left(15 p_{6}^{4}+15 p_{6}^{2} \varphi_{2}-\varphi_{2}^{4}\right)+p_{3}\left(6 p_{6}^{5}-20 p_{6}^{3} \varphi_{2}^{2}+6 p_{6} \varphi_{2}^{4}\right)\right)\right), \\
& \varphi_{k+1}=\cos \left[\beta a_{1}\right] \varphi^{k}-\beta^{2} \sin \left[\beta a_{1}\right] C_{10}^{k+1} C_{10}^{k} .
\end{aligned}
$$

In equations (11) there are denoted:

$$
\cos \left[a_{1} \varphi_{1}\right]=p_{1}, \cos \left[a_{1} \varphi_{2}\right]=p_{3}, \sin \left[a_{1} \varphi_{1}\right]=p_{2}, \sin \left[a_{1} \varphi_{2}\right]=4, \log \left[\rho_{1}\right]=p_{5}, \log \left[\rho_{2}\right]=p_{6}
$$

By revising of $(k-1)$ area we accept the origin of coordinates on its end and direct thexaxle to the opposite side. Then for it $f_{1}(x)$ and $f_{2}(x)$ will have the same form as on the $k$ area, and arbitrary constants (let's denote them as $C_{i}^{k-1}$ ) will be determined from (11), if we change in them $\eta_{k+1}, \mu_{k+1}, \varsigma_{k+1}, \xi_{k+1}, \varphi_{k+1}$, by $\eta_{k-1}, \mu_{k-1}, \varsigma_{k-1}, \xi_{k-1}, \varphi_{k-1}$.

Conditions on line of $k$ edge taking into account different directions of axes $x$ for contiguous areas that are received from variation equation we will write in form:

$$
\begin{aligned}
& \frac{\partial^{4}}{\partial x^{3} \partial y}\left(F-\frac{B h}{G_{\varsigma}} \nabla^{2} F\right)_{x=+0}+\frac{\partial^{4}}{\partial x^{3} \partial y}\left(F-\frac{B h}{G_{\zeta}} \nabla^{2} F\right)_{x=-0}= \\
& =\frac{B_{p k}}{\bar{B}}\left[\frac{\partial^{5}}{\partial x^{4} \partial y}\left(F-\frac{B h}{G_{\varsigma}} \nabla^{2} F\right)-\mu \frac{\partial^{5}}{\partial x^{2} \partial y^{3}}\left(F-\frac{B h}{G_{\zeta}} \nabla^{2} F\right)\right]_{x=0} ; \\
& \int\left[\frac{\partial^{4}}{\partial x^{2} \partial y^{2}}\left(F-\frac{B h}{G_{3}} \nabla^{2} F\right)-\mu \frac{\partial^{4}}{\partial x^{4}}\left(F-\frac{B h}{G_{3}} \nabla^{2} F\right)\right]_{x=+0} d x= \\
& =-\int\left[\frac{\partial^{4}}{\partial x^{2} \partial y^{2}}\left(F-\frac{B h}{G_{3}} \nabla^{2} F\right)-\mu \frac{\partial^{4}}{\partial x^{4}}\left(F-\frac{B h}{G_{3}} \nabla^{2} F\right)\right]_{x=-0} ; \\
& \left(\frac{\partial}{\partial x} \nabla^{4} F+\frac{\partial \Psi}{\partial y}\right)_{x=+0}=-\left(\frac{\partial}{\partial x} \nabla^{4} F+\frac{\partial \Psi}{\partial y}\right)_{x=-0} ; \\
& \left(\frac{\partial}{\partial x} \nabla^{6} F\right)_{x=+0}+\left(\frac{\partial}{\partial x} \nabla^{6} F\right)=-\left\{\frac{D_{p k}}{D^{*}}\left(1-\frac{B h}{G_{3}} \nabla^{2}\right) \frac{\partial^{4}}{\partial y^{4}} \nabla^{4} F+\right. \\
& \left.+\frac{B_{p k}}{\bar{B}} \frac{\bar{B}}{D^{*} R^{2}}\left[\frac{\partial^{4}}{\partial x^{4}}\left(F-\frac{B h}{G_{3}} \nabla^{2} F\right)-\mu \frac{\partial^{4}}{\partial x^{2} \partial y^{2}}\left(F-\frac{B h}{G_{3}} \nabla^{2} F\right)\right]-\left(1-\frac{B h}{G_{3}} \nabla^{2}\right) \frac{P_{P}}{D^{*}} \nabla^{4} F\right\}_{x=0} ; \\
& {\left[-\frac{\partial \Psi}{\partial y}+\frac{B h}{G_{3}} \frac{\partial}{\partial y} \nabla^{6} F\right]_{x=+0}=0 .} \\
& \text { Here } \gamma=\frac{D_{p k} \pi^{4}}{b^{4} D^{*}} .
\end{aligned}
$$

Substituting into system (12) solution of equations (6) and (7), we will have the system of equations, where values of arbitrary constants $C_{i}^{k-1}$ and $C_{i}^{k+1}$ are determined by expressions (11). 
Solution of this system we will found in form:

$$
\eta_{k}=A \sin \frac{k \pi}{m} ; \mu_{k}=B \sin \frac{k \pi}{m} ; \varsigma_{k}=C \sin \frac{k \pi}{m} ; \xi_{k}=M \sin \frac{k \pi}{m} ; \varphi_{k}=E \sin \frac{k \pi}{m} .
$$

Unknown $\eta_{k}, \mu_{k}, \varsigma_{k}, \xi_{k}, \varphi_{k}$, which are included into this system must satisfy conditions of solution periodicity:

$$
\eta_{0}=\mu_{0}=\varsigma_{0}=\xi_{0}=\varphi_{0}=\eta_{m}=\mu_{m}=\varsigma_{m}=\xi_{m}=\varphi_{m}=0 .
$$

Equating determinant, which consists of coefficients at $A, B, C, M$, $E$, to zero we will get frequency equation of three-layered sloping cylindrical shell, which is supported by regular transverse ribs, at edge simple support.

Parameter of the first frequency we will find by solving the transcendent frequency equation. In the tables 1-4 there are given values of parameter of first frequency of free vibrations $m_{\omega}$ of sloping cylindrical shell, that is supported by one and three ribs. Value $m_{\omega}$ was determined with taking into account the Reisner's edge effect (top row of tables) and without it (lower row of tables).If we put in the frequency equation $k_{0}=0$, we will get the frequency equation for one-layered supported shell (with bending stiffness $D^{*}=2 B H^{2}$ ).

\begin{tabular}{|c|c|c|c|c|c|c|c|c|c|}
\hline \multirow{3}{*}{$\frac{a}{b}$} & \multicolumn{3}{|c|}{$\gamma=1 \alpha^{2}=5$} & \multicolumn{3}{|c|}{$\gamma=2 \alpha^{2}=5$} & \multicolumn{3}{|c|}{$\gamma=3 \quad \alpha^{2}=5$} \\
\hline & \multicolumn{3}{|c|}{$\mathrm{k}_{0}$} & \multicolumn{3}{|c|}{$\mathrm{k}_{0}$} & \multicolumn{3}{|c|}{$\mathrm{k}_{0}$} \\
\hline & 0,2 & 0,3 & 0,4 & 0,2 & 0,3 & 0,4 & 0,2 & 0,3 & 0,4 \\
\hline \multirow{2}{*}{1,0} & 7,591 & 6,969 & 6,434 & 7,591 & 6,969 & 6,434 & 7,591 & 6,969 & 6,434 \\
\hline & 7,588 & 6,961 & 6,424 & 7,585 & 6,958 & 6,421 & 7,584 & 6,956 & 6,421 \\
\hline \multirow{2}{*}{2,0} & 7,591 & 6,969 & 6,434 & 7,591 & 6,969 & 6,434 & 7,591 & 6,969 & 6,434 \\
\hline & 7,578 & 6,953 & 6,411 & 7,576 & 6,947 & 6,410 & 7,575 & 6,947 & 6,409 \\
\hline \multirow{2}{*}{3,0} & 7,591 & 6,969 & 6,434 & 7,591 & 6,969 & 6,434 & 7,591 & 6,969 & 6,434 \\
\hline & 7,570 & 6,942 & 6,399 & 7,570 & 6,940 & 6,396 & 7,567 & 6,935 & 6,395 \\
\hline
\end{tabular}

TABLE 1. Values of parameter $m_{\omega}$ for shell, which is supported by one rib

\begin{tabular}{|c|c|c|c|c|c|c|c|c|c|}
\hline \multirow{3}{*}{$\frac{a}{b}$} & \multicolumn{3}{|c|}{$\gamma=1 \alpha^{2}=10$} & \multicolumn{3}{|c|}{$\gamma=2 \alpha^{2}=10$} & \multicolumn{3}{|c|}{$\gamma=3 \quad \alpha^{2}=10$} \\
\hline & \multicolumn{3}{|c|}{$\mathrm{k}_{0}$} & \multicolumn{3}{|c|}{$\mathrm{k}_{0}$} & \multicolumn{3}{|c|}{$\mathrm{k}_{0}$} \\
\hline & 0,2 & 0,3 & 0,4 & 0,2 & 0,3 & 0,4 & 0,2 & 0,3 & 0,4 \\
\hline \multirow{2}{*}{1,0} & 7,082 & 6,458 & 5,923 & 7,082 & 6,458 & 5,923 & 7,082 & 6,458 & 5,923 \\
\hline & 7,047 & 6,418 & 5,881 & 7,042 & 6,413 & 5,876 & 7,037 & 6,408 & 5,878 \\
\hline \multirow{2}{*}{2,0} & 7,082 & 6,458 & 5,923 & 7,082 & 6,458 & 5,923 & 7,082 & 6,458 & 5,923 \\
\hline & 7,000 & 6,365 & 5,823 & 6,991 & 6,356 & 5,813 & 6,983 & 6,345 & 5,801 \\
\hline \multirow{2}{*}{3,0} & 7,082 & 6,458 & 5,923 & 7,082 & 6,458 & 5,923 & 7,082 & 6,458 & 5,923 \\
\hline & 6,965 & 6,330 & 5,775 & 6,965 & 6,324 & 5,775 & 6,955 & 6,320 & 5,770 \\
\hline
\end{tabular}

TABLE 2. Values of parameter $m_{\omega}$ for shell, which is supported by one rib

TABLE 3. Values of parameter $m_{\omega}$ for shell, which is supported by three rib

\begin{tabular}{|c|ccc|ccc|ccc|}
\hline \multirow{2}{*}{$\frac{3}{b}$} & \multicolumn{3}{|c|}{$\gamma=1 \alpha^{2}=5$} & \multicolumn{3}{c|}{$\gamma=2 \alpha^{2}=5$} & \multicolumn{3}{c|}{$\gamma=3 \alpha^{2}=5$} \\
\cline { 2 - 10 } & \multicolumn{3}{|c|}{$\mathrm{k}_{0}$} & \multicolumn{3}{c|}{$\mathrm{k}_{0}$} & \multicolumn{3}{c|}{$\mathrm{k}_{0}$} \\
\cline { 2 - 10 } & 0,2 & 0,3 & 0,4 & 0,2 & 0,3 & 0,4 & 0,2 & 0,3 & 0,4 \\
\hline \multirow{2}{*}{1,0} & 7,594 & 6,969 & 6,433 & 7,594 & 6,969 & 6,433 & 7,594 & 6,969 & 6,433 \\
& 7,589 & 6,961 & 6,426 & 7,588 & 6,961 & 6,427 & 7,586 & 6,957 & 6,429 \\
2,0 & 7,594 & 6,969 & 6,433 & 7,594 & 6,969 & 6,433 & 7,594 & 6,969 & 6,433 \\
& 7,581 & 6,972 & 6,418 & 7,582 & 6,951 & 6,417 & 7,577 & 6,951 & 6,410 \\
3,0 & 7,594 & 6,969 & 6,433 & 7,594 & 6,969 & 6,433 & 7,594 & 6,969 & 6,433 \\
& 7,572 & 6,942 & 6,409 & 7,578 & 6,946 & 6,398 & 7,568 & 6,937 & 6,396 \\
\hline
\end{tabular}


TABLE 4 . Values of parameter $m_{\omega}$ for shell, which is supported by three rib

\begin{tabular}{|c|ccc|ccc|ccc|}
\hline \multirow{3}{*}{$a$} & \multicolumn{3}{|c|}{$\gamma=1 \alpha^{2}=10$} & \multicolumn{3}{c|}{$\gamma=2 \alpha^{2}=10$} & \multicolumn{3}{c|}{$\gamma=3 \alpha^{2}=10$} \\
\cline { 2 - 10 }$b$ & \multicolumn{3}{|c|}{$\mathrm{k}_{0}$} & \multicolumn{3}{c|}{$\mathrm{k}_{0}$} & & \multicolumn{3}{c|}{$\mathrm{k}_{0}$} \\
& 0,2 & 0,3 & 0,4 & 0,2 & 0,3 & 0,4 & 0,2 & 0,3 & 0,4 \\
\hline \multirow{2}{*}{1,0} & 7,083 & 6,456 & 5,924 & 7,083 & 6,456 & 5,924 & 7,083 & 6,456 & 5,924 \\
& 7,062 & 6,439 & 5,897 & 7,087 & 6,433 & 5,886 & 7,077 & 6,442 & 5,910 \\
2,0 & 7,083 & 6,456 & 5,924 & 7,083 & 6,456 & 5,924 & 7,083 & 6,456 & 5,924 \\
& 7,058 & 6,436 & 5,895 & 7,079 & 6,415 & 5,878 & 7,088 & 6,426 & 5,874 \\
3,0 & 7,083 & 6,456 & 5,924 & 7,083 & 6,456 & 5,924 & 7,083 & 6,456 & 5,924 \\
& 7,043 & 6,419 & 5,874 & 7,064 & 6,412 & 5,863 & 7,057 & 6,402 & 5,849 \\
\hline
\end{tabular}

\section{Conclusion}

Thus, a computational model is constructed and an algorithm for investigating free vibrations of a threelayered sloping cylindrical shell is developed, which is supported by transverse ribs.

Analyzing the value of parameter of the first frequency of free vibrations $m_{\omega}$ we should note that support of shell by transverse ribs has no significant influence on free vibrations frequency. At shear parameter $\mathrm{k}_{0}$ increase the frequency of free vibrations reduces. At free vibrations frequencies determination, the Reisner's edge effect has no significant influence at any value of shear parameter.

\section{REFERENCES}

[1] Grigolyuk E.I. (1973) Ustoychivost i kolebaniya trehsloynykh obolochek. Moscow: Mashinostroeniye, 172 pp.

[2] Grugolyuk E.I. (1988) Mnogosloyniye armirovanniye obolochki: Raschet pnevmaticheskih shin. Moscow: Mashinostroeniye, 288 pp.

[3] Popov B.G. (1993) Raschet mnogosloynikh konstrukciy variatsionno-matrichnymi metodami: Uchebnoye posobiye. Moscow: Izdatelstvo MGTU, $294 \mathrm{pp}$.

[4] Bolotin V.V. (1980) Mekhanika mnogosloynikh konstrukciy. Moscow: Mashinostroeniye, 376 pp.

[5] Filippov S.B. (2007) Kolebaniya tonkoy trekhsloynoy cilindricheskoy obolochki. Vestnik SPbGU, 2(1): 150-156.

[6] Starovoytov E.I. (2013) Kolebaniya trekhsloynikh cilidricheskih obolochek v uprugoy srede Vinklera pri rezonanse. Mekhanika mashin, mekhanizmov i materialov. 4(25): 70-73.

[7] Solomonov Y.S. (2009) Metody rascheta cilindricheskih obolochek iz kompozitsionnykh materialov. Moscow: FIZMATLIT, 264 pp.

[8] Babich D.V. O chastotnom spektre kolebaniy neodnorodnykh cilindricheskih obolochek. Prikladnaya mekhanika. 10 (31): 67-72.

[9] Latanskaya L.A. (2012) Matematicheskoye modelirovaniye dinamiki vinyzhdennykh kolebaniy konstruktivno neodnorodnykh obolochek vrascheniya. Problemy obchyslyvalnoyi mekhaniky i micnosti konstrukciy. 19: 205-211.

[10] Yemelianova T.A. (2002) Differencialnye uravneniya svobodnykh kolebaniy trekhsloynoy obolochki, podkreplennoy rebrami zhestkosti. Teoreticheskaya i prikladnaya mekhanika. Sbornik nauchnykh trudov. Minsk: UP “Tehnoprint”, 169-181.

[11] Yemelianova T.A. (2016) Rozvyazuyuche rivnyannya vilnykh kolyvan trisharovoyi cilindrichnoyi obolonky, yaka pidkriplena rebramy zhorstkosti. Visnyk Khersonskogo natsionalnogo tekhnichnogo universytetu. 2(57): 11-17.

[12] Kyrychenko V.L. (2007) Vilni kolyvannya trisharovoyi polohoyi cilindrichnoyi paneli z lehkym zapovnyuvachem, yaka pidkriplena pryamoliniynymy rebramy zhorstkosti. Proceeding of $5^{\text {th }}$ International scientific and practical Internet-conference. Poltava. 24-28. 